\title{
Endogenous Nitric Oxide Protects against Platelet-Activating Factor-Induced Bowel Injury in the Rat
}

\author{
WILLIAM MACKENDRICK, MICHAEL CAPLAN, AND WEI HSUEH \\ Department of Pediatrics, Evanston Hospital, Evanston. Illinois 60201, and the Department of Pathology. \\ Children's Memorial Hospital, Northwestern University Medical School, Chicago, Illinois 60614
}

\begin{abstract}
Platelet-activating factor (PAF) causes bowel necrosis in animal models that is histologically identical to that seen in neonatal necrotizing enterocolitis, but little is known about endogenous mechanisms that might protect against PAF-induced bowel injury. We hypothesized that endogenous nitric oxide might represent such a protective mechanism. Adult male Sprague-Dawley rats were pretreated with $2.5 \mathrm{mg} / \mathrm{kg} \mathrm{N}^{\mathbf{G}}$-nitro-L-arginine methyl ester (L-NAME), a potent nitric oxide synthase inhibitor, and given injections of $1.5 \mu \mathrm{g} / \mathrm{kg}$ PAF $15 \mathrm{~min}$ later. Animals treated with normal saline placebo, LNAME alone, and PAF alone were also studied. Superior mesenteric artery blood flow and blood pressure were continuously recorded. At the end of $2 \mathrm{~h}$ or upon death of the animal, hematocrit was measured and intestinal samples were taken for histologic examination and determination of myeloperoxidase activity, a measure of intestinal neutrophil content. Compared with animals given PAF alone, animals pretreated with L-NAME followed by PAF developed significantly worse bowel injury (median injury scores: 2.5 versus $0.5, p=0.005$ ), hemoconcentration (final hematocrit $65.2 \pm 2.0 \%$ versus $53.9 \pm 1.0 \%, p<0.001$ ), and intestinal myeloperoxidase activity $(12.45 \pm 1.94 \mathrm{U} / \mathrm{g}$ versus $6.51 \pm 0.57 \mathrm{U} / \mathrm{g}, p<0.01$ ). The last two effects were further accentuated when $10 \mathrm{mg} / \mathrm{kg}$ L-NAME was given before PAF. Treatment with sodium nitroprusside, a nitric oxide donor, for 10 min before and after PAF administration reversed the effects of L-NAME. Animals pretreated with phenylephrine rather than L-NAME did not develop worse injury than animals treated with PAF alone despite comparable reductions in superior mesenteric blood flow before PAF treatment. Treatment with superoxide dismutase and catalase did not ameliorate the effects of $\mathrm{L}$ NAME on PAF-induced injury, hemoconcentration, and neutrophil infiltration into the bowel. We conclude that endogenous nitric oxide defends against PAF-induced bowel injury by antagonizing certain immediate effects of PAF, particularly those leading to capillary leakage and neutrophil infiltration into the bowel. Our studies also suggest that the mechanism of action does not involve vasodilatation or scavenging of oxygen radicals. (Pediatr Res 34: 222-228, 1993)
\end{abstract}

\section{Abbreviations}

NO, nitric oxide

PAF, platelet-activating factor

SOD, superoxide dismutase

CAT, catalase

Received January 7, 1993; accepted March 4, 1993.

Correspondence and reprint requests: William MacKendrick, M.D., Department of Pediatrics, Evanston Hospital, 2650 Ridge Ave., Evanston, IL. 60201.

\author{
NS, normal saline \\ L-NAME, $\mathbf{N}^{\mathrm{G}}$-nitro-L-arginine methyl ester \\ L-NMMA, $\mathbf{N}^{\mathrm{G}}$-monomethyl-L-arginine \\ MPO, myeloperoxidase \\ MAP, mean arterial pressure \\ SMA, superior mesenteric artery \\ SNP, sodium nitroprusside \\ PHENYL, L-phenylephrine
}

PAF is a potent phospholipid inflammatory mediator that has been implicated in the pathogenesis of neonatal necrotizing enterocolitis in both animal models (1) and human studies (2). Neutrophil margination and capillary leakage are associated with the development of PAF-induced bowel injury (1). In the intact animal, mesenteric vasoconstriction caused by secondary mediators may also be important $(3,4)$. A key initiating event in the pathogenesis of PAF-induced bowel injury may be neutrophil activation, because rats and mice rendered leukopenic with vinblastine are relatively protected from PAF-induced bowel injury $(5,6)$.

NO is continuously produced in the vascular endothelium by the constitutive NO synthase enzyme using $L$-arginine as a substrate (7). NO is now generally accepted to be the equivalent of endothelium-derived relaxing factor and appears to play an important role in maintaining baseline vasodilator tone (7). NO has also recently been shown to play a role in maintaining microvascular integrity both under normal physiologic conditions (8) and in response to inflammatory mediators (9). Leukocyte adherence to the vascular endothelium also appears to be modulated by NO under normal conditions (10); however, modulation of leukocyte adherence by NO under conditions of tissue injury and inflammation has not been explored. The mechanisms by which NO modulates microvascular integrity and leukocyte adherence are not completely understood. However, Kubes et al. (8) have speculated that scavenging of superoxide anions by NO may be involved, at least under normal conditions (10).

Little is presently known about endogenous mechanisms that might defend against PAF-induced bowel injury in the intact animal. As a promoter of microvascular integrity, inhibitor of leukocyte adherence, and vasodilator, endogenous NO has several biologic properties that suggest that it might constitute such a defense mechanism. Therefore, the present study was designed to determine if endogenous NO protects against PAF-induced bowel injury in a whole animal model. We also sought to explore the mechanisms responsible for such an effect.

\section{MATERIALS AND METHODS}

Animals. Young adult male Sprague-Dawley rats (Harlan Sprague-Dawley, Inc., Indianapolis, IN) weighing 120 to $200 \mathrm{~g}$ were 
used in all experiments. Animals were fasted overnight but were allowed free access to drinking water before the experiments. All procedures performed on live animals were approved by the Institutional Animal Care and Use Committee.

Surgical preparation. Anesthesia was induced by pentobarbital injection. $60 \mathrm{mg} / \mathrm{kg}$ intraperitoneally. A tracheostomy was performed to ensure maintenance of a stable airway, and the left carotid artery and right jugular vein were catheterized to allow blood pressure monitoring, blood sampling, and drug administration. The left femoral vein was also catheterized to permit concomitant infusion of two drugs if required. The abdomen was opened along the midline, and a short segment of the SMA was carefully isolated near its origin from the aorta. An ultrasonic flow probe (Transonics Systems, Inc., Ithaca, NY) was cuffed around the SMA to permit continuous monitoring of SMA flow, and the abdomen was closed. Rectal temperature was maintained between 35.5 and $37.0^{\circ} \mathrm{C}$ throughout the experiment with an electric heating pad. After surgical preparation, the animals were allowed to stabilize for $30 \mathrm{~min}$ before the start of the experiment.

Experimental protocols. The first series of protocols was designed to determine the effect of NO synthase inhibition on PAFinduced bowel injury, hemoconcentration, and neutrophil infiltration into the intestinal wall. To inhibit NO production, we used L-NAME (Sigma Chemical Co., St. Louis, MO), an arginine analog that is a potent inhibitor of NO synthase (11). At the start of each experiment $(-15 \mathrm{~min})$, animals received an i.v. bolus injection of either NS placebo (control and PAF groups), 2.5 $\mathrm{mg} / \mathrm{kg} \mathrm{L}-\mathrm{NAME}$ in 120 to $200 \mu \mathrm{L}$ NS (2.5-L-NAME and $2.5-\mathrm{L}-$ NAME+PAF groups), or $10 \mathrm{mg} / \mathrm{kg} \mathrm{L}$-NAME in 120 to $200 \mu \mathrm{L}$ NS (10-L-NAME+PAF group). Fifteen min later (time 0), they received another bolus injection of either NS placebo (control and 2.5-L-NAME groups) or $1.5 \mu \mathrm{g} / \mathrm{kg}$ PAF (1-O-alkyl-2-acetylsn-glyceryl-3-phosphorylcholine; Sigma) in 150 to $250 \mu \mathrm{L}$ of PBS solution containing $0.5 \%$ BSA (PAF, 2.5-L-NAME+PAF and 10-L-NAME+PAF groups).

The second set of protocols was designed to explore the mechanism of L-NAME - induced accentuation of the bowel injury produced by PAF. To confirm that the effects of L-NAME were due to inhibition of NO production, rats in the SNP+LNAME+PAF group were injected with $2.5 \mathrm{mg} / \mathrm{kg} \mathrm{L-NAME}$ and PAF as described above and also received a continuous i.v. infusion of SNP (Sigma), an NO donor, from -10 to $+10 \mathrm{~min}$ via a syringe pump. The duration of nitroprusside infusion was limited to $10 \mathrm{~min}$ before and after PAF administration because the half-life of PAF is very short in plasma (12), and we reasoned that the immediate effects of PAF should be essential to subsequent production of bowel injury and, under our hypothesis, should also be antagonized by NO. The dosage of nitroprusside was titrated for each animal to normalize the MAP after LNAME administration without causing hypotension and ranged from 2.0 to $3.0 \mu \mathrm{g} / \mathrm{kg} / \mathrm{min}$ delivered in a volume of 5.7 to 8.4 $\mu \mathrm{L} / \mathrm{min}$. To determine if the effects of $\mathrm{L}-\mathrm{NAME}$ could be attributed to mesenteric vasoconstriction, rats in the PHENYL+PAF group received PAF as described above and also received a continuous infusion of PHENYL (Sigma) at $35 \mathrm{mg} / \mathrm{kg} / \mathrm{h}$ from -15 to $+30 \mathrm{~min}$ in a volume of $5.7 \mu \mathrm{L} / \mathrm{min}$ via syringe pump. This dose was chosen after preliminary experiments revealed that it produced a reduction in SMA flow similar to that caused by $2.5 \mathrm{mg} / \mathrm{kg}$ L-NAME. The duration of PHENYL infusion was chosen after initial experiments revealed that SMA blood flow in the 2.5-L-NAME group was not significantly different from controls by $30 \mathrm{~min}$. Finally, the importance of $\mathrm{NO}$ as an oxygen radical scavenger in this model was assessed in the SOD/CAT $+\mathrm{L}$ NAME+PAF group by administering bovine erythrocyte SOD (4200 U/mg; Sigma) and bovine liver CAT (48 $700 \mathrm{U} / \mathrm{mg}$; Sigma) to animals treated with $2.5 \mathrm{mg} / \mathrm{kg} \mathrm{L-NAME}$ and PAF as described above. A bolus dose of $42000 \mathrm{U} / \mathrm{kg}$ SOD and 420000 $\mathrm{U} / \mathrm{kg} \mathrm{CAT}$ was given at $-10 \mathrm{~min}$, followed by continuous infusion of $2100 \mathrm{U} \mathrm{SOD}$ and $21000 \mathrm{UCAT} / \mathrm{kg} / \mathrm{min}$ from -10 to $+10 \mathrm{~min}$ in a volume of $8.4 \mu \mathrm{L} / \mathrm{min}$. This dosing interval was chosen to correspond to the period of SNP infusion in the SNP+L-NAME+PAF group.

In all groups, animals were studied for $2 \mathrm{~h}$ after PAF/placebo administration or until death. Arterial blood samples for hematocrit determination were obtained at the beginning and end of the experiment. The MAP and SMA flows were continuously recorded using a four-channel chart recorder (MFE, Inc., Salem, $\mathrm{NH})$. At the end of the experiment, the distal half of the small intestine was removed and immediately washed and flushed with ice-cold NS. A representative segment was preserved in $10 \%$ formalin solution for later histologic examination, and the remainder of the specimen was snap-frozen in liquid nitrogen and stored at $-70^{\circ} \mathrm{C}$ for future determination of intestinal MPO activity by the method of Krawisz et al. (13).

Histologic examination. Formalin-preserved intestinal samples were embedded in paraffin, sectioned with a microtome, and stained with hematoxylin and eosin stain for microscopic examination. Specimens were reviewed in a blinded manner by two independent observers, and injury was graded on a numerical scale. Grade 0 corresponded to specimens with minimal or no injury, grade 1 to specimens with epithelial cell loss from the villi, grade 2 to specimens with partial loss or necrosis of the villi, and grade 3 to specimens with loss or necrosis of the entire mucosa. When the assigned injury grade disagreed, the average of the two grades was used.

Statistical analysis. Analysis of variance and $t$ test were used to compare two or more groups of normally distributed data. whereas the Mann-Whitney U test was used to compare groups of nonparametric data. All values are reported as the mean \pm SEM. A $p$ value of 0.05 was considered significant in all comparisons.

\section{RESULTS}

Mortality. No mortality occurred among animals in the PAF, PHENYL+PAF, or 2.5-L-NAME groups. In contrast, four of seven animals in the 2.5-L-NAME+PAF group and five of five animals in the 10-L-NAME+PAF group died before the end of the experimental period (Fig. 1). All animals in the 10-LNAME+PAF group died within 25 min after PAF administration. SNP infusion completely prevented the mortality associated with L-NAME+PAF administration. In the SOD/CAT+LNAME+PAF group, three of six animals died, but the survival times among nonsurvivors were significantly longer compared with the $2.5-\mathrm{L}-\mathrm{NAME}+\mathrm{PAF}$ group $(73.0 \pm 4.4 \mathrm{~min}$ versus $20.0 \pm 5.5 \mathrm{~min}, p<0.001)$.

Intestinal injury. Median intestinal injury grades for all groups are summarized in Figure 1. Grade 1 intestinal injury was seen in some specimens in the control group, possibly due to preparation artifact. No injury was seen in the 2.5-L-NAME group, and only grade- 0 to -1 injury occurred in the PAF group (Fig. $2 A$ ). However, grade- 2 to -3 injury was consistently seen in the 2.5-L-NAME+PAF group ( $p=0.005$ versus PAF group). The injury seen in the 2.5 -L-NAME+PAF group typically consisted of coagulation necrosis, with partial or total loss of the villi (Fig. $2 B$ ). The degree of injury was comparable in the $2.5-\mathrm{L}-$ NAME+PAF and 10-L-NAME+PAF groups. However, it should be noted that all animals in the 10-L-NAME+PAF group died within $25 \mathrm{~min}$ of PAF administration, limiting the time available for injury to progress. Injury tended to be less severe in the SNP+L-NAME+PAF group $(p=0.07$ versus $2.5-\mathrm{L}-$ NAME+PAF) but not in the SOD/CAT+L-NAME+PAF group. Injury in the PHENYL+PAF group was no worse than in the PAF group.

Blood pressure. MAP data are summarized in Figure 3. Baseline MAP were comparable among all groups. Administration of $2.5 \mathrm{mg} / \mathrm{kg}$ L-NAME at $-15 \mathrm{~min}$ (2.5-L-NAME and $2.5-\mathrm{L}-$ NAME+PAF groups) caused a significant increase in MAP by time 0 . Animals in the $2.5-\mathrm{L}-\mathrm{NAME}$ group were significantly hypertensive when compared with controls through $90 \mathrm{~min}$. In 


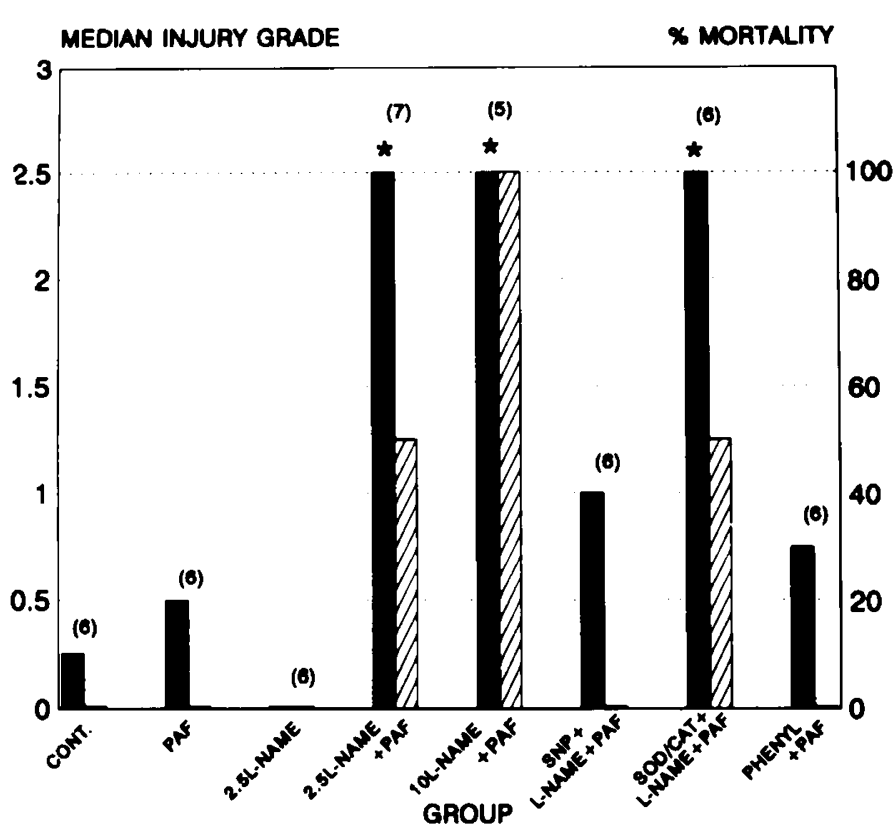

Fig. 1. Median microscopic intestinal injury scores (black bars) and percentage of mortality ( hatched bars) in each of the experimental groups. Details of the intestinal injury scoring system are given in the text. Numbers in parentheses indicate the number of animals in each experimental group. Group names reflect the compounds administered to each group as detailed in the text. ${ }^{*}, p<0.05$ for injury score compared with PAF group.

the 10-L-NAME+PAF group (MAP data not shown), the MAP increased by $3.04 \pm 0.79 \mathrm{kPa} 15 \mathrm{~min}$ after $10 \mathrm{mg} / \mathrm{kg}$ L-NAME, similar to the response seen with $2.5 \mathrm{mg} / \mathrm{kg} \mathrm{L-NAME}$. In the SNP+L-NAME+PAF group, the MAP had returned to baseline by time 0 . Animals in the PHENYL+PAF group tended to experience an increase in MAP greater than that seen in animals treated with $2.5 \mathrm{mg} / \mathrm{kg} \mathrm{L-NAME}$ by time 0 ( $p=0.07$ versus 2.5 L-NAME group).

Bolus injection of PAF at time 0 caused the MAP to drop to 4 to $5 \mathrm{kPa}$ within $30 \mathrm{~s}$ in all animals so treated (data at $30 \mathrm{~s}$ not shown). In the PAF and PHENYL+PAF groups, animals then experienced a gradual recovery throughout the remainder of the experiment, with MAP not significantly different from controls by $60 \mathrm{~min}$. Among survivors in the $2.5-\mathrm{L}-\mathrm{NAME}+\mathrm{PAF}$ group, the MAP recovery after PAF injection was generally similar to that seen in the PAF group, whereas nonsurvivors and all animals in the 10-L-NAME+PAF group demonstrated little or no improvement in MAP before death. Animals in the SNP+LNAME+PAF group demonstrated significantly better MAP recovery after PAF injection than did animals in the PAF and 2.5. $\mathrm{L}-\mathrm{NAME}+\mathrm{PAF}$ groups. Animals in the SOD/CAT+LNAME+PAF group had MAP similar to those in the $2.5-\mathrm{L}-$ NAME+PAF group at all time points (data not shown).

SMA blood flow. The SMA blood flow data are summarized in Figure 4. Animals in the 2.5-L-NAME and 2.5-L-NAME+PAF groups experienced significant reductions in SMA blood flow by time 0 . A comparable degree of blood flow reduction was seen in animals given $10 \mathrm{mg} / \mathrm{kg} \mathrm{L-NAME}$ (data not shown). Although animals in the 2.5-L-NAME group subsequently tended to have lower SMA flow than controls, the difference was not significant by $30 \mathrm{~min}$. Animals in the SNP+L-NAME+PAF group demonstrated partial recovery of SMA blood flow by time 0 . PHENYL infusion caused a reduction in SMA flow comparable to that caused by $2.5 \mathrm{mg} / \mathrm{kg}$ L-NAME by time 0 .

PAF injection caused the SMA flow to drop to 10 to $20 \%$ of baseline within $30 \mathrm{~s}$, concomitant with the fall in MAP, in all animals so treated (data at $30 \mathrm{~s}$ not shown). Despite a gradual recovery in MAP, animals in the PAF group had significantly lower SMA flows than controls through $90 \mathrm{~min}$. SMA flows in PHENYL+PAF animals after PAF injection were similar to those in the PAF group. Surviving animals in the 2.5-LNAME+PAF group had significantly lower SMA flows through the end of the experiment than animals in the PAF and $2.5-\mathrm{L}-$ NAME groups, despite recovery of MAP. After PAF injection, animals in the SNP+L-NAME+PAF group had SMA flows that tended to be better than those in the 2.5-L-NAME+PAF group, but the difference did not reach statistical significance at any time. SMA flows in the SOD/CAT+L-NAME+PAF group (data not shown) were similar to those in the 2.5-L-NAME+PAF group at all time points.

Hematocrit and MPO data. The final hematocrit and intestinal MPO activity data are summarized in Figure 5. Initial hematocrits were similar among all groups, with an overall average value of $49.9 \pm 1.3 \%$. Final hematocrits were similar among the control, 2.5-L-NAME, and PAF groups, although the final hematocrit tended to be higher in the PAF group. Final hematocrit was significantly higher in the 2.5-L-NAME+PAF group $(65.2 \pm$ $2.0 \%$ versus $53.9 \pm 1.0 \%$ in the PAF group, $p<0.001$ ). This effect was dependent on the dose of L-NAME, because the final hematocrit was even higher among animals in the 10-LNAME+PAF group $(72.0 \pm 1.8 \%, p=0.046$ versus $2.5-\mathrm{L}-$ NAME+PAF group). The increase in final hematocrit seen in the 2.5-L-NAME+PAF group was prevented in the SNP+LNAME+PAF group but not in the SOD/CAT+L-NAME+PAF group. Final hematocrit among animals in the PHENYL+PAF group was similar to that in the PAF group.

Animals in the PAF group had significantly more intestinal MPO activity than controls $(6.51 \pm 0.57 \mathrm{U} / \mathrm{g}$ versus $1.88 \pm 0.08$ $\mathrm{U} / \mathrm{g}, p=0.02$ ), whereas the 2.5-L-NAME group was similar to controls. Animals in the 2.5-L-NAME+PAF group had significantly more intestinal MPO activity than the PAF group $(12.45 \pm 1.94 \mathrm{U} / \mathrm{g}, p<0.01)$, and activity was even higher in the 10-L-NAME+PAF group $(23.79 \pm 4.20 \mathrm{U} / \mathrm{g}, p=0.02$ versus 2.5-L-NAME+PAF). The increase in intestinal MPO activity was completely reversed in the SNP+L-NAME+PAF group but was unaffected in the SOD/CAT+L-NAME+PAF group. Activity was not increased in the PHENYL+PAF group compared with the PAF group.

\section{DISCUSSION}

The pathogenesis of PAF-induced bowel injury is complex and incompletely understood. A key initiating event may be neutrophil activation, adherence, and migration into intestinal tissues, accompanied by capillary leakage. Subsequently, secondary vasoactive mediators, such as leukotrienes, may be released, causing local tissue hypoxia and worsening injury. The interaction of these factors with endogenous protective mechanisms may determine the extent of intestinal injury in any given instance, but very little is currently known about such protective mechanisms.

Using a whole-animal model, we have shown that endogenous NO constitutes a major defense mechanism against PAF-induced bowel injury. Administration of the potent NO synthase inhibitor, L-NAME, at doses of 2.5 and $10 \mathrm{mg} / \mathrm{kg}$, markedly worsened the bowel injury seen after administration of $1.5 \mu \mathrm{g} / \mathrm{kg}$ PAF. This finding cannot be attributed to an independently toxic effect of L-NAME, because animals treated with $2.5 \mathrm{mg} / \mathrm{kg} \mathrm{L-NAME}$ alone did not develop injury. Provision of exogenous NO in the form of SNP partially reversed the injurious effects of L-NAME, supporting the conclusion that inhibition of endogenous NO production was largely responsible for accentuation of PAFinduced bowel injury.

Our results are in general agreement with those reported by other investigators who have explored the protective effects of NO in different models of gastrointestinal injury. Using a model of ethanol-induced gastritis in the rat, MacNaughton et al. (14) demonstrated that NO protects against gastric mucosal injury. 


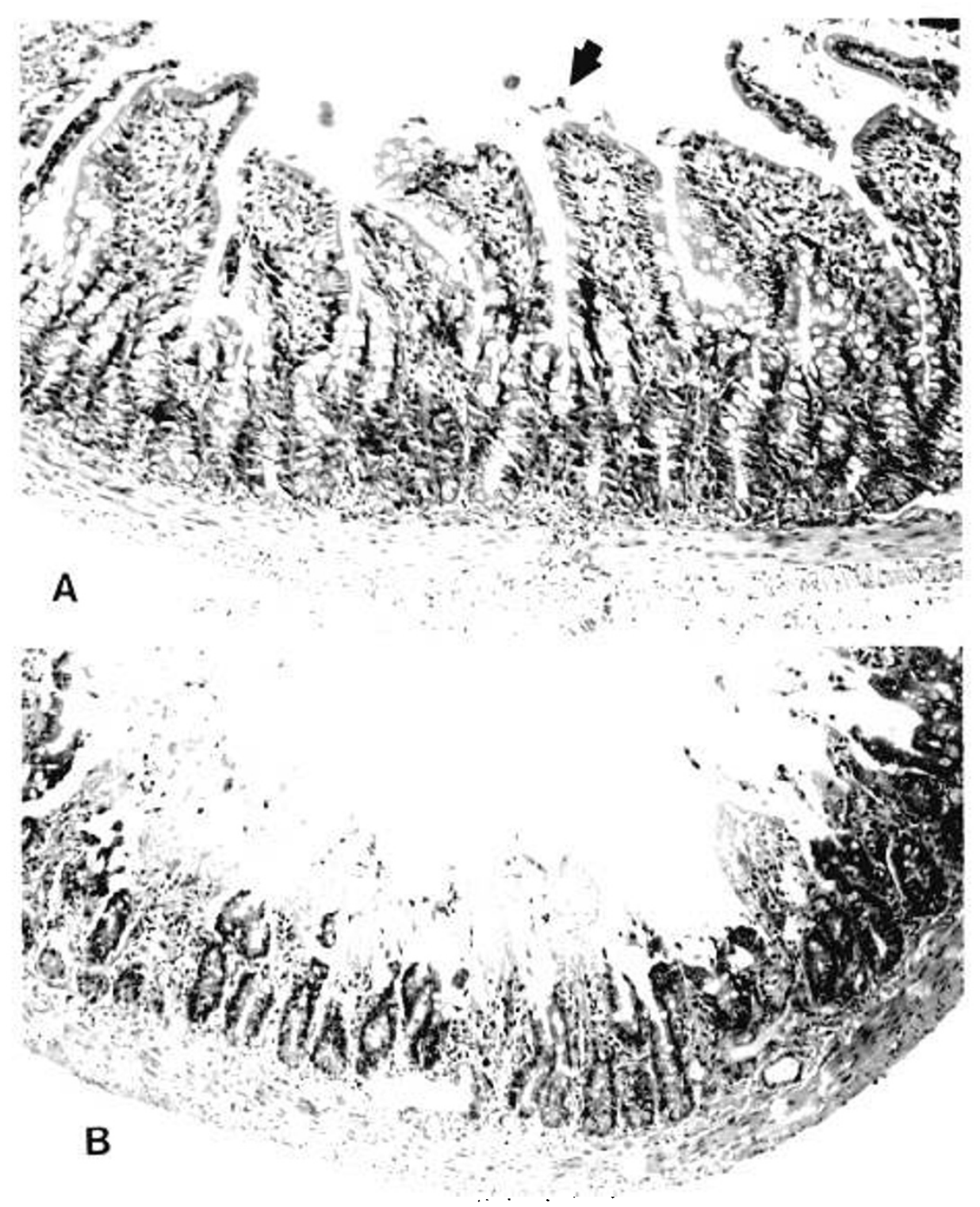

Fig. 2. Photomicrographs of intestinal injury seen in PAF-treated animals $(A)$ and in animals treated with $2.5 \mathrm{mg} / \mathrm{kg} \mathrm{l}-\mathrm{NAME}+\mathrm{PAF}(B)$. PAFtreated animals typically demonstrated only mild epithelial cell loss from the villi (arrow), whereas animals given 2.5-1.-NAME+PAF often developed coagulation necrosis of the villi, with partial or total loss of the villi (original magnification $\times 120$ ).

Kubes (15) found that inhibition of endogenous NO synthesis worsened intestinal ischemia-reperfusion injury in the cat. Similarly, Carey et al. (16) and Aoki et al. (17) have reported that administration of exogenous NO lessens the degree of shock and tissue injury in other models of intestinal ischemia-reperfusion injury. Inhibition of endogenous NO synthesis has also been reported to worsen endotoxin-induced intestinal injury in the rat (18). Because PAF has been implicated in the pathogenesis of both ischemia-reperfusion injury (19) and endotoxin-induced intestinal injury (20), our results may help to explain those reported by the latter investigators.

However, our results stand in contrast to those reported by Boughton-Smith et al. (21), who found that inhibition of endogenous NO production by the arginine analog L-NMMA did not clearly accentuate PAF-induced gastrointestinal injury in the rat. This difference in results is probably due to a difference in potency of the NO synthase inhibitors used, inasmuch as LNAME is reported to be a 100 -fold more potent inhibitor of the endothelial cell NO synthase than is L-NMMA (11). Indeed, in preliminary experiments, we were also unable to document a consistent effect of L-NMMA on PAF-induced intestinal injury (unpublished data).
Our model. in which PAF was administered i.v., may not completely mimic the interaction between the effects of PAF and endogenous NO under normal phýsiologic conditions, because much of the PAF synthesized by stimulated leukocytes and endothelial cells remains intracellular and may function as an autocrine mediator (22). However, elevated circulating levels of PAF have been documented in several diseases, including neonatal necrotizing enterocolitis (2), suggesting that, in certain pathophysiologic states, PAF may act as an endocrine mediator through interaction with its receptors on target cell types.

In addition to its effects on intestinal injury, L-NAME also increased mortality rates in PAF-treated rats in a dose-dependent manner that was reversed by SNP. Nonsurvivors in the two LNAME+PAF groups all died after periods of prolonged hypotension. Because cardiopulmonary function was not an outcome variable of primary interest in our study, we can only speculate about the cause of death in these animals. PAF is known to have marked negative inotropic effects (23), and inhibition of endogenous NO production is known to decrease cardiac output in the intact rat (24). Therefore, I-NAME and PAF could have synergistically caused severe impairment of cardiac function in some animals, leading to death. Among survivors in the $2.5-\mathrm{L}$ - 

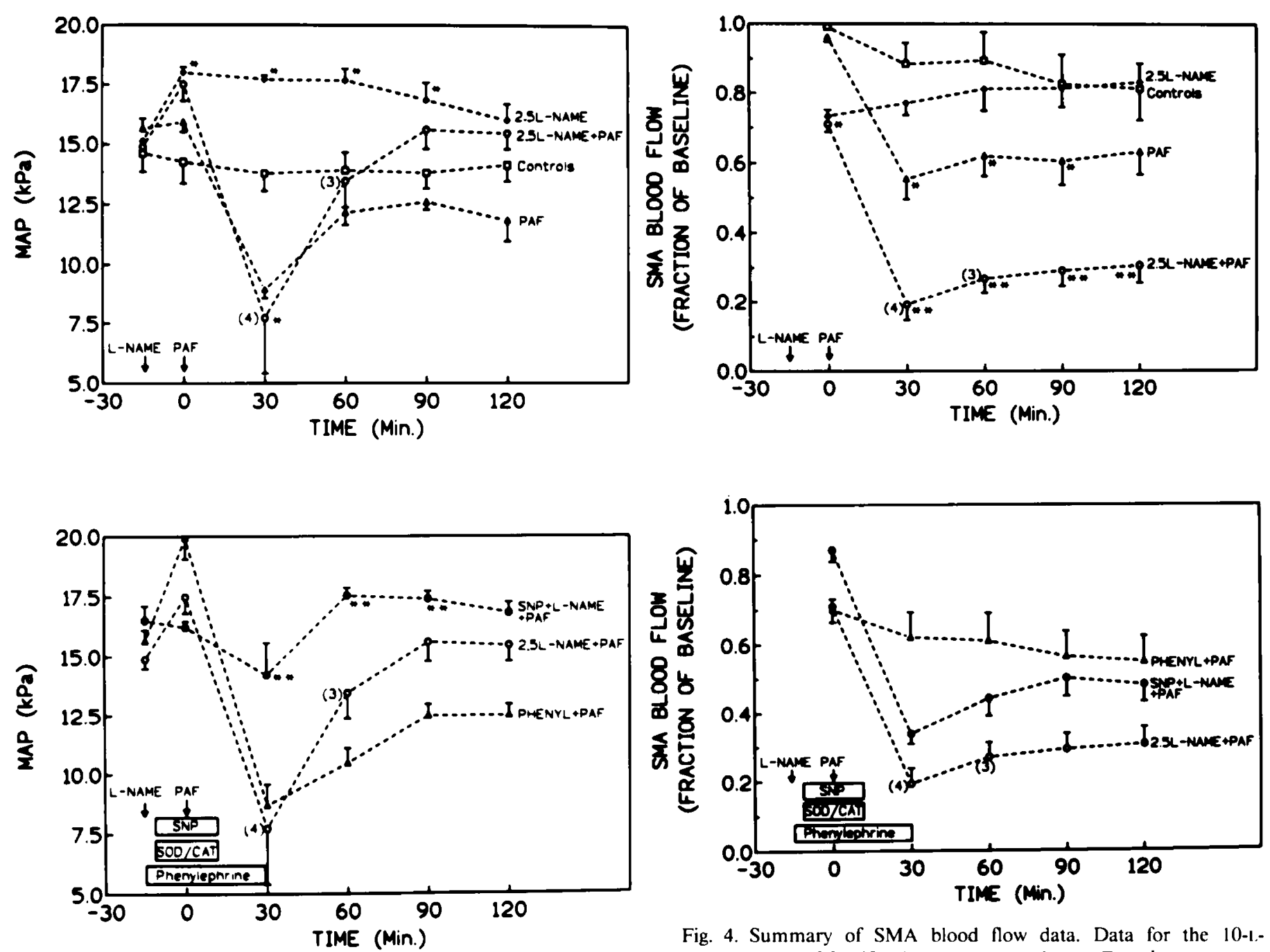

Fig. 4. Summary of SMA blood flow data. Data for the 10-1.-

Fig. 3. Summary of MAP data. Data for the 10-L-NAME+PAF and SOD/CAT groups are not shown. Error bars represent \pm SEM. For the 2.5-L-NAME+PAF group, numbers in parentheses indicate number of animals surviving after deaths occurred. In the lower panel, enclosed boxes indicated the duration of infusion of the indicated compound, whereas the arrows indicate the timing of bolus doses of L-NAME and PAF. Group names reflect the compounds administered to that group as detailed in the text (e.g. animals in the PHENYL+PAF group received an infusion of PHENYL from -15 to +30 min and an injection of PAF at $0 \mathrm{~min}$, but did not receive L-NAME). ${ }^{*}, p<0.05$ compared with controls; ${ }^{* *}, p<0.05$ compared with 2.5 -L-NAME+PAF group.

NAME+PAF group, the MAP had returned to normal by 60 min, but SMA flows remained significantly reduced through the end of the study period. Therefore, intestinal hypoperfusion in the 2.5-L-NAME+PAF group during the last hour of the study can be attributed to mesenteric vasoconstriction rather than systemic hypotension.

The pronounced mesenteric vasoconstriction seen in the 2.5L-NAME+PAF group could have contributed to intestinal injury in those animals. However, the cause of this marked vasoconstriction is unclear. Given alone, L-NAME produced significant mesenteric vasoconstriction compared with controls only for the first $30 \mathrm{~min}$, whereas marked vasoconstriction was present in the 2.5-L-NAME+PAF group through the end of the experiment. In addition, infusion of phenylephrine at a dose duplicating the severity and duration of vasoconstriction produced by L-NAME alone failed to accentuate PAF-induced injury. Therefore, it seems unlikely that removal of the baseline vasodilator tone provided by NO was responsible for either the persistent mesNAME+PAF and SOD/CAT groups are not shown. Error bars represent \pm SEM. For the 2.5-1.-NAME+PAF group, numbers in parentheses indicate number of animals surviving after deaths occurred. In the lower panel, the enclosed boxes and arrow's indicate the timing of infusion of the indicated compound as explained in Figure 3. Group names reflect the compounds administered to that group as detailed in the text. ${ }^{*}, p<$ 0.05 compared with controls: ${ }^{* *}, p<0.05$ compared with PAF group.

enteric vasoconstriction or the injury seen in the $2.5-\mathrm{L}$ NAME+PAF group. Instead, the mesenteric vasoconstriction seen in the 2.5-L-NAME+PAF group may have reflected an exacerbation of the in vivo vasoconstrictor effects of PAF. However, this is not likely to have been the primary mechanism of injury, inasmuch as correction of mesenteric hypoperfusion in PAF-treated rats does not reliably prevent intestinal injury (4).

We found that PAF-induced hemoconcentration and neutrophil infiltration into the bowel were also augmented by L-NAME in a dose-dependent manner that was reversed by nitroprusside. These findings are consistent with the view that endogenous NO may promote microvascular integrity and inhibit leukocyte adherence and emigration, thereby limiting PAF-induced bowel injury. Previous observations $(5,25)$, as well as the present study, have shown a positive correlation between neutrophil infiltration into the bowel and development of intestinal injury. Neutrophil infiltration and vascular leakage appear to occur very soon after PAF administration and probably precede the development of bowel injury, because animals in the 10-L-NAME+PAF group. none of which survived longer than $25 \mathrm{~min}$ after PAF administration, had markedly elevated final hematocrits and intestinal MPO activity.

The hypertensive effects of L-NAME when given alone at a 
Final Hct (\%)

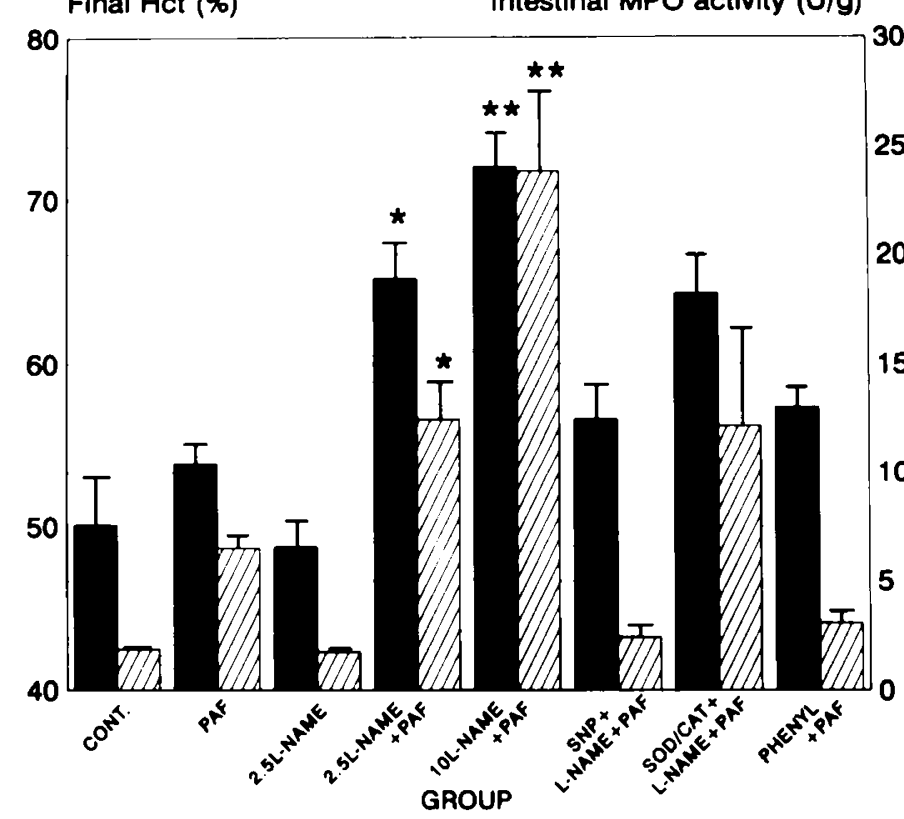

Fig. 5. Final hematocrit (black bars) and intestinal MPO activity (hatched bars). Error bars represent \pm SEM. Group names reflect the compounds administered to that group as detailed in the text. ${ }^{*}, p<$ 0.05 compared with PAF group; ${ }^{* *}, p<0.05$ compared with $2.5-\mathrm{L}-$ NAME+PAF group.

dose of $2.5 \mathrm{mg} / \mathrm{kg}$ persisted through $90 \mathrm{~min}$, suggesting a relatively long period of NO synthase inhibition by this compound. However, provision of exogenous NO as nitroprusside for the 10 min preceding and after PAF administration was sufficient to substantially reverse the effects of L-NAME on PAF-induced bowel injury, hemoconcentration, and neutrophil infiltration into the bowel wall. This observation suggests that the immediate effects of PAF are essential for the initiation and subsequent development of bowel injury and that these effects are antagonized by NO.

Superoxide production by PAF-activated neutrophils in vitro has been shown to reach maximal levels within 5 min of PAF administration (26). Because superoxide appears to be involved in PAF-induced leukocyte adherence and capillary leakage (27), one might expect that inactivation of superoxide anions by NO constitutes an important defense mechanism against the immediate effects of PAF, which later lead to bowel injury. However, our data do not support this conclusion. Treatment with large doses of SOD and CAT for $10 \mathrm{~min}$ before and after PAF administration did not ameliorate the bowel injury, hemoconcentration, or neutrophil infiltration seen in animals pretreated with L-NAME. This finding suggests that scavenging of superoxide anion is not an important mechanism by which NO prevents initiation of PAF-induced bowel injury. However, our findings do not preclude the possibility that NO could limit PAFinduced bowel injury by acting as an oxygen radical scavenger after injury has already been initiated, because oxygen radicals are known to be ultimately important in the production of PAFinduced bowel injury (28). Also, nonsurvivors in the SOD/ CAT+L-NAME+PAF group survived significantly longer than nonsurvivors in the 2.5-L-NAME+PAF group, suggesting that superoxide inactivation by NO may play a role in ameliorating early PAF-induced systemic toxicity.

Prevention of PAF-induced neutrophil activation by endogenous NO could account for the results of the present study. This hypothesis is supported by a recent report from Clancy $e t$ al. (29). Using an in vitro system, these investigators found that the neutrophilic NADPH oxidase is directly inhibited by NO via effects on the membrane portion of the enzyme before activation. This enzyme is an important source of superoxide anions in
PAF-activated neutrophils. Therefore, inhibition of endogenous NO before PAF administration could result in excessive superoxide production in response to PAF, leading to accentuation of PAF-induced bowel injury. Such exuberant production of superoxide could have exceeded the scavenging capacity of the doses of SOD and CAT given in the SOD/CAT+1.-NAME+PAF group or may have persisted beyond the duration of SOD and CAT administration. Under this hypothesis, injury would have been prevented in the SNP+L-NAME+PAF group because NO was replaced before PAF administration, thereby inhibiting neutrophilic NADPH oxidase activation by PAF.

Finally, our findings may have some impact on proposed new approaches to the treatment of septic shock. A growing body of evidence suggests that the hypotension seen in endotoxin- and cytokine-induced shock may be mediated by excessive NO production $(30,31)$. These findings have prompted some investigators to suggest that NO synthase inhibitors such as L-NAME may be beneficial in the treatment of septic shock, and the use of LNAME to treat refractory hypotension in patients with septic shock has been reported in the literature (32). However, PAF is known to be an important mediator of septic shock (33) and also appears to mediate the bowel injury produced by endotoxin (20) and tumor necrosis factor- $\alpha(34)$. Because our results suggest that endogenous NO is important in defending against PAF-induced bowel injury and mortality, extreme caution should be used when contemplating administration of NO synthase inhibitors to patients with septic shock.

\section{REFERENCES}

1. Hsueh W. Gonzalez-Crussi F 1988 Ischemic bowel necrosis induced by plateletactivating factor: an experimental model. Meth Achiev Exp Pathol 13:208239

2. Caplan MS, Sun X-M. Hsueh W. Hageman JR 1990 Role of platelet activating factor and tumor necrosis factor-alpha in neonatal necrotizing enterocolitis. J Pediatr 1 16:960-964

3. Hsueh W. Gonzalez-Crussi F. Arroyave J 1986 Platelet-activating factorinduced ischemic bowel necrosis: an investigation of secondary mediators in its pathogenesis. Am J Pathol 122:231-239

4. Zhang C. Hsueh W 1991 PAF-induced bowel necrosis: effects of vasodilators. Dig Dis Sci 36:634-640

5. Musemeche C, Caplan M, Hsueh W. Sun X. Kelly A 1991 Experimental necrotizing enterocolitis: the role of polymorphonuclear neutrophils. J Pediatr Surg 26:1047-1050

6. Sun XM. Hsueh W 1991 Platelet-activating factor produces shock, in vivo complement activation, and tissue injury in mice. J Immunol 147:509-514

7. Moncada S, Palmer RMJ, Higgs EA 1991 Nitric oxide: physiology, pathophysiology and pharmacology. Pharmacol Rev 43:109-142

8. Kubes P. Granger DN 1992 Nitric oxide modulates microvascular permeability. Am J Physiol 262:H611-H615

9. Filep JG, Foldes-Filep E, Sirois P 1993 Nitric oxide modulates vascular permeability in the rat coronary circulation. $\mathrm{Br} \mathrm{J}$ Pharmacol 108:323-326

10. Kubes P, Suzuki M, Granger DN 1991 Nitric oxide: an endogenous modulator of leukocyte adhesion. Proc Natl Acad Sci USA 88:4651-4655

11. Gross SS, Stuehr DJ, Aisaka K. Jaffe EA. Levi R, Griffith OW 1990 Macrophage and endothelial cell nitric oxide synthesis: cell-type selective inhibition by NG-aminoarginine, NG-nitroarginine and NG-methylarginine. Biochem Biophys Res Commun 170:96-103

12. Blank ML, Cress EA, Whittle T, Snyder F 1981 In vivo metabolism of a new class of biologically active phospholipids: 1-alkyl-2-acetyl-sn-glycero-3-phosphocholine, a platelet activating hypotensive phospholipid. Life Sci 29:769775

13. Krawisz JE, Sharon P. Stenson WF 1984 Quantitative assay for acute intestinal inflammation based on myeloperoxidase activity. Gastroenterology 87:13441348

14. MacNaughton WK, Cirino G, Wallace JL 1989 Endothelium-derived relaxing factor (nitric oxide) has protective actions in the stomach. Life Sci 45:18691876

15. Kubes $P 1993$ Ischemia-reperfusion in feline small intestine: a role for nitric oxide. Am J Physiol 264:G143-G149

16. Carey C, Siegfried MR, Ma XJ, Weyrich AS, Lefer AM 1992 Antishock and endothelial protective actions of a NO donor in mesenteric ischemia and reperfusion. Circ Shock 38:209-214

17. Aoki N, Johnson G, Lefer AM 1990 Beneficial effects of two forms of NO administration in feline splanchnic artery occlusion shock. Am J Physiol 258:G275-G281

18. Hutcheson IR, Whittle BJR, Boughton-Smith NK 1990 Role of nitric oxide in maintaining vascular integrity in endotoxin-induced acute intestinal damage in the rat. Br J Pharmacol 101:815-820

19. Filep J, Herman F, Braquet P. Mozes T 1989 Increased levels of platelet 
activating factor in blood following intestinal ischemia in the dog. Biochem Biophys Res Commun 158:353-359

20. Hsueh W, Gonzalez-Crussi F. Arroyave JL 1987 Platelet-activating factor: an endogenous mediator for bowel necrosis in endotoxemia. FASEB J 1:403405

21. Boughton-Smith NK, Deakin AM, Whittle BJR 1992 Actions of nitric oxide on the acute gastrointestinal damage induced by PAF in the rat. Agents Actions (special conference issue):C3-C9

22. Stewart AG, Dubbin PN, Harris T, Dusting GJ 1990 Platelet-activating factor may act as a second messenger in the release of icosanoids and superoxide anions from leukocytes and endothelial cells. Proc Natl Acad Sci USA 87:3215-3219

23. Levi R, Burke J, Guo Z-G, Hattori Y, Hoppens CM, McManus LM, Hanahan DJ, Pinckard RN 1984 Acetyl glyceryl ether phosphorylcholine (AGEPC): a putative mediator of cardiac anaphylaxis in the guinea pig. Circ Res 54:117124

24. Loeb A, Longnecker D 1992 Inhibition of endothelium-derived relaxing factordependent circulatory control in intact rats. Am J Physiol 262:H1494H 1500

25. Zhang C. Hsueh W. Caplan MS, Kelly A 1991 Platelet activating factorinduced shock and intestinal necrosis in the rat: role of endogenous plateletactivating factor and effect of saline infusion. Crit Care Med 19:1067-1072

26. Shaw J, Pinckard RN, Ferrigni K. McManus L. Hanahan DJ 1981 Activation of human neutrophils with 1-O-hexadecyl/octadecyl-2-acetyl-sn-glyceryl-3phosphorylcholine (platelet activating factor). J Immunol 127:1250-1255

27. Kubes P, Suzuki M, Granger DN 1990 Modulation of PAF-induced leukocyte adherence and increased microvascular permeability. Am J Physiol 259:G859-G864

28. Cueva JP. Hsueh W 1988 Role of oxygen derived free radicals in platelet activating factor induced bowel necrosis. Gut 29:1207-1212

29. Clancy RM, Leszczynska-Piziak J, Abramson S 1992 Nitric oxide, an endothelial cell relaxation factor, inhibits neutrophil superoxide anion production via a direct action on the NADPH oxidase. J Clin Invest 90:1116-1121

30. Thiemermann C. Vane J 1990 Inhibition of nitric oxide synthesis reduces the hypotension induced by bacterial lipopolysaccharides in the rat in vivo. Eur J Pharmacol 182:591-595

31. Kilbourn RG, Gross SS, Jubran A, Adams J, Griffith OW, Levi R, Lodato RF $1990 \mathrm{~N}^{\mathrm{G}}$-methyl-L-arginine inhibits tumor necrosis factor-induced hypotension: implications for the involvement of nitric oxide. Proc Natl Acad Sci USA 87:3629-3632

32. Petros A, Bennett D, Vallance $P 1991$ Effect of nitric oxide synthase inhibitors on hypotension in patients with septic shock. Lancet 338:1557-1558

33. Doebber TW, Wu MS, Robbins JC, Choy BM, Chang MN, Shen TY 1985 Platelet activating factor (PAF) involvement in endotoxin-induced hypotension in rats: studies with PAF-receptor antagonist kadsurenone. Biochem Biophys Res Commun 127:799-808

34. Sun XM. Hsueh W 1988 Bowel necrosis induced by tumor necrosis factor in rats is mediated by platelet-activating factor. J Clin Invest 81:1328-1331 